\title{
La deposición de cenizas volcánicas modula la descomposición de hojarasca en bosques de Nothofagus dombeyi del norte de Patagonia
}

\author{
M. Victoria Piazza ${ }^{12, \mathbb{1} \otimes}$; Thomas Kitzberger ${ }^{3} \&$ Enrique J. Chaneton $^{1}$ \\ ${ }^{1}$ Universidad de Buenos Aires, Consejo Nacional de Investigaciones Científicas y Técnicas, Instituto de Investigaciones \\ Fisiológicas y Ecológicas Vinculadas a la Agricultura (IFEVA), Facultad de Agronomía, Buenos Aires, Argentina. ${ }^{2}$ Cátedra \\ de Dasonomía, Facultad de Agronomía, Buenos Aires, Argentina. ${ }^{3}$ Laboratorio Ecotono, Instituto de Investigaciones en \\ Biodiversidad y Medioambiente (INIBIOMA), CONICET y Universidad Nacional del Comahue, San Carlos de Bariloche.
}

Resumen. Los disturbios generados por erupciones volcánicas son parte de la dinámica natural de los ecosistemas. La formación de suelo y el reciclado de nutrientes dependen de la descomposición tanto de la materia orgánica enterrada bajo las cenizas como de la broza vegetal aportada luego del disturbio. Este trabajo examinó la descomposición de hojarasca en bosques de coihue (Nothofagus dombeyi) afectados por la deposición de cenizas emitidas en 2011 por el complejo Volcán Puyehue-Cordón Caulle en la Patagonia. El estudio incluyó dos sitios a diferentes distancias del volcán, con distinta cantidad de cenizas y precipitación anual (1600-1900 mm/año). En cada sitio se marcaron dos parcelas, una con presencia y otra con ausencia de pastoreo de vacunos ( $>50$ años). En cada parcela se determinó la pérdida de masa de hojarasca de coihue luego de un año de incubación, en tres posiciones ( $\mathrm{n}=8$ bloques/parcela): sobre suelo bajo cenizas, sobre suelo sin cenizas y sobre las cenizas. La hojarasca bajo cenizas se descompuso un $74 \%$ más rápido en el sitio más húmedo que en el más seco. La descomposición fue más lenta sobre la capa de cenizas que sobre el suelo orgánico, y ese efecto fue más evidente en el sitio más cercano al volcán que en el más alejado (19\% vs. $9 \%$ ). La descomposición sobre el suelo fue equivalente en los tratamientos con y sin la capa superficial de cenizas. La descomposición fue menor en parcelas con vs. sin pastoreo, pero la influencia de pastoreo no modificó las diferencias de descomposición entre posiciones sobre cenizas vs. sobre el suelo. Los resultados muestran que la comunidad de descomponedores se mantiene activa en suelos de bosques con deposición de cenizas, lo que contribuiría a mantener el suministro de nutrientes para la vegetación luego de la erupción volcánica.

[Palabras clave: disturbios naturales, erupciones volcánicas, funcionamiento del ecosistema, gradiente ambiental, pastoreo histórico]

\begin{abstract}
Aвstract. Volcanic ash deposition modulates leaf-litter decomposition in Nothofagus dombeyi forests of NW Patagonia. Disturbances produced by volcanic eruptions are part of natural ecosystem dynamics. Soil formation and nutrient cycling depend on the decomposition both of organic matter buried under the ashes and on the litter produced after the disturbance. Here we evaluated leaf litter decomposition in Nothofagus dombeyi (coihue) forests affected by massive ash deposition from the 2011 eruption of the Volcán Puyehue-Cordón Caulle complex in NW Patagonia, Argentina. The study comprised two sites at different distances from the volcano, with different amounts of ash and annual precipitation (1600-1900 mm/year). In each site, two plots were delimited, with and without long-term livestock grazing (>50 years). N. dombeyi litter mass loss after one year was estimated in three positions ( $\mathrm{n}=8$ blocks/plot): on organic soil and under the ash layer, on organic soil after removal of ashes, and on top of the ash layer. Leaf litter beneath the ash layer was decomposed $74 \%$ faster in the wetter site than in the drier site. Decomposition was generally slower onto the ash layer surface than on the organic soil, and this effect was stronger in the wetter site near the volcano than in the driest and farthest site (19\% vs. $9 \%)$. Litter decomposition on the soil layer was equivalent to treatments with or without the ash surface. Decomposition was slower in livestock-occupied plots than in livestock-free plots, but livestock history did not affect litter decomposition of ash topped soil vs. lower soil organic layers. Our results show that decomposer communities remain active in forest soils under ash-fall layers, thus maintaining nutrient supply to vegetation after volcanic eruptions.
\end{abstract}

[Keywords: natural disturbances, volcanic eruptions, ecosystem functioning, environmental gradient, historical grazing] 


\section{INTRODUCCIÓN}

Los disturbios naturales constituyen una parte integral de la dinámica de los ecosistemas. Los agentes de disturbio afectan distintos niveles de organización, que pueden moldear propiedades del suelo, condiciones ambientales y comunidades bióticas (Pickett et al. 1989; Gutschick and BassiriRad 2003), así como al flujo de energía y reciclado de nutrientes. En particular, los disturbios que provocan las erupciones volcánicas suelen afectar áreas geográficas extensas y ambientes físicos y bióticos heterogéneos (Dale et al. 2005). El impacto de una erupción varía desde eventos catastróficos de deposición de lava y la iniciación de una sucesión primaria, hasta eventos menos destructivos en zonas alejadas del cráter donde se deposita material piroclástico (del Moral and Grishin 1999; Veblen et al. 2016). En este último caso, las alteraciones sobre la vegetación y el ambiente por deposición de cenizas suelen desencadenar diferentes respuestas de los organismos persistentes (Zobel and Antos 1997; Turner et al. 1998; Dale et al. 2005; Magnin et al. 2016). Es esperable entonces que las funciones ecosistémicas se regeneren con diferente rapidez, según la posición del sitio a lo largo de un gradiente de impacto por la deposición de cenizas (Hopkins et al. 2007; Chaneton et al. 2014). Mas aún, los efectos ambientales o antrópicos que influyen en distintos aspectos del ecosistema podrían interactuar con los efectos locales de las cenizas (Turner and Gardner 2015).

Los suelos de origen volcánico suelen asociarse con áreas fértiles por su capacidad de intercambio de nutrientes, almacenamiento de agua y materia orgánica (Dahlgren et al. 2004). La deposición de cenizas altera las propiedades bióticas y abióticas del entorno y modifica las condiciones para la descomposición de hojarasca enterrada luego de la erupción (Edmonds and Erickson 1994; Berenstecher et al. 2016). Además de la necromasa enterrada, la dinámica de nutrientes del suelo también depende de los aportes de hojarasca producida por la vegetación remanente y / o restablecida luego del disturbio (Edmonds and Erickson 1994; Hopkins et al. 2007). Pueden existir variaciones en la descomposición que ocurre sobre un sustratoinerteaportadoporlascenizas o en contacto con el suelo orgánico subyacente (Edmonds and Erickson 1994; Hopkins et al. 2007; Castellano et al. 2015). Por ejemplo, luego de la erupción del volcán Santa Elena (Washington, EEUU) se observó en bosques remanentes que la tasa de descomposición de hojarasca sobre las cenizas fue hasta dos veces más lenta respecto a la hojarasca enterrada bajo cenizas y sobre el suelo orgánico (Edmonds and Erickson 1994; Erickson and Edmonds 1994). Dichas diferencias se relacionarían a la mayor humedad del suelo enterrado bajo las cenizas. Además, el efecto resultante sobre los procesos del suelo dependerá del espesor y la composición química de las cenizas, con distintas consecuencias para la persistencia y regeneración de la flora y la fauna (Dale et al. 2005; Veblen et al. 2016). Pese a la importancia de este fenómeno, hasta el momento hay poca información cuantitativa sobre la dinámica de la descomposición en distintas capas del suelo en bosques afectados por sucesivas erupciones volcánicas (Turner et al. 1998; Edmonds and Erickson 1994; Erickson and Edmonds 1994).

Los cambios del ecosistema en respuesta a la erupción volcánica reflejan el contexto ambiental que opera a escala regional o local (Edmonds and Erickson 1994; Zobel and Antos 1997; Chaneton et al. 2014). La variación preexistente de factores regionales, como la humedad y temperatura o la abundancia y diversidad de niveles tróficos (Semmartin et al. 2004; Kardol and Wardle 2010), pueden amortiguar o exacerbar los efectos de las cenizas, por ejemplo,al modificar la producción foliar del dosel del bosque (Chaneton et al. 2014). Además, factores locales como la estructura del sotobosque podrían facilitar el restablecimiento del funcionamiento del suelo luego de una erupción. La introducción de grandes herbívoros altera la estructura, composición y el micro-ambiente del sotobosque, con variadas consecuencias sobre los procesos del suelo (Wardle and Bardgett 2004; Kardol and Wardle 2010; Piazza et al. 2016). Por ejemplo, en bosques templados de Japón los cambios ambientales provocados por el tránsito de ciervos desaceleraron la tasa de descomposición de la hojarasca, en comparación con la exclusión del pastoreo (Suzuki and Ito 2014; Kasahara et al. 2016). La deposición de cenizas volcánicas podría tener impactos ambientales similares a los generados por grandes herbívoros, al modificar la estructura del sotobosque, la temperatura micro-climática o la dinámica del agua y los nutrientes del suelo (Wardle and Bardgett 2004; Edmonds and Erickson 1994). Sin embargo, el impacto inicial de la deposición de cenizas, y sus consecuencias ecológicas, podrían ser más severos en bosques no afectados por ganado introducido que en sitios impactados por el pastoreo 
extensivo (cf. Piazza et al. 2016). Por último, la falta de conocimiento sobre la interacción entre ambos disturbios para la regeneración del funcionamiento del bosque sugiere la necesidad de estudiar posibles sinergismos o antagonismos (Pickett et al. 1989) entre las cenizas volcánicas y la historia de pastoreo.

Los bosques Andino-Patagónicos presentan disturbios de origen volcánico cada 50 a 100 años (Singer et al. 2008; Iglesias et al. 2011; Veblen et al. 2016). En junio de 2011, la erupción del complejo Volcán Puyehue-Cordón Caulle (CVPCC) liberó mil millones de toneladas de cenizas que cubrieron gran parte de las provincias de Neuquén y Río Negro (Gaitán et al. 2011). El evento provocó la muerte de árboles en pie en un radio de unos $15 \mathrm{~km}$ del volcán (Magnin et al. 2016); fuera de esa zona, las cenizas cubrieron el suelo y la vegetación en una gran extensión (Veblen et al. 2016). Estudios recientes evidenciaron algunos de los cambios ecológicos inducidos por las cenizas. Se registraron cambios en el crecimiento radial de los árboles, la productividad foliar y los niveles de herbivoría del dosel (Chaneton et al. 2014; Magnin et al. 2016). Las cenizas alteraron las propiedades físico-químicas del suelo, que incluyeron una mayor capacidad de retención hídrica y un menor contenido de nutrientes (Cremona et al. 2011; González et al. 2015; Berenstecher et al. 2016). Los efectos en los organismos del suelo implicaron tanto reducciones en la abundancia de artrópodos y detritívoros (Elizalde 2014; Oleiro 2015), como la estimulación de la actividad de descomponedores (Berenstecher etal. 2016). En bosques donde se depositaron cenizas finas, la descomposición fue más rápida en la hojarasca enterrada en el suelo orgánico que en aquella depositada sobre las cenizas (Oleiro 2015). Finalmente, en un mesocosmos experimental se observó una mayor descomposición de la hojarasca bajo cenizas finas que la incubada sobre el suelo sin cenizas (Berenstecher et al. 2016). A pesar de estas evidencias, aún no está claro cómo la deposición de cenizas controla la descomposición de hojarasca en diferentes posiciones del suelo y en distintos tipos ambientales de bosque (Erickson and Edmonds 1994).

El objetivo de este trabajo fue examinar la influencia de la deposición masiva de cenizas emitidas por el CVPCC sobre la descomposición de hojarasca en bosques de Nothofagus dombeyi (coihue) del NO de la Patagonia. Se evaluaron los efectos de la posición de la broza en el perfil vertical cenizas/suelo y del contexto ambiental determinado por la distancia del bosque al volcán y la presencia histórica de ganado vacuno (Piazza et al. 2016). En particular, evaluamos las siguientes predicciones:

(1) la hojarasca caída sobre las cenizas se descompone más lentamente que la hojarasca enterrada bajo la capa de cenizas y en contacto con el suelo orgánico;

(2) la magnitud de las diferencias entre la descomposición la hojarasca sobre vs. bajo la capa de cenizas es mayor en bosques cercanos al volcán, por su mayor cantidad de cenizas, en relación con los bosques más alejados del volcán que recibieron menores cantidades de cenizas;

(3) las diferencias entre la descomposición de hojarasca sobre vs. bajo la capa de cenizas son más evidentes en bosques cuya estructura y composición del sotobosque no fueron modificados por el pastoreo extensivo de ganado.

\section{MATERIALES Y MÉTODOS}

\section{Sitio de estudio}

El estudio se localizó en bosques dominados por coihue, dentro del Parque Nacional Nahuel Huapi, provincia de Neuquén. El área fue afectada por la deposición de cenizas del CVPCC en junio de 2011, que cubrieron completamente el suelo formando capas de entre 5 y $30 \mathrm{~cm}$ de espesor (Gaitán et al. 2011). Según estudios locales, la composición mineralógica del material depositado fue uniforme y similar a los productos emitidos por el mismo sistema en eventos anteriores, con dominancia de vidrio volcánico $(70 \%)$ y escasos aluminosilicatos (Cremona et al. 2011). Las cenizas depositadas en el área de S. C. de Bariloche tuvieron un $\mathrm{pH}=6.5$, baja conductividad eléctrica (0.18, susp. 1:2.5), bajo contenido de nutrientes $(0.02 \% \mathrm{C}, 0.003 \% \mathrm{~N}$, $3.1 \mathrm{ppm}$ P) y buena capacidad de retención hídrica ( $12.7 \%$ capacidad de campo) (Cremona et al. 2011).

Se seleccionaron dos sitios con bosques maduros de coihue (200-300 años de edad), con la mayor similitud posible en cuanto a la cobertura del dosel, la influencia lacustre y la historia de incendios antiguos (Piazza et al. 2016). Los sitios estudiados se encontraron a diferentes distancias del volcán y presentaron variaciones importantes en la cantidad y 
granulometría del material depositado por la erupción (Gaitán et al. 2011). La posición geográfica de los bosques determina distintos niveles de precipitación y temperatura media anuales (PMA, TMA) (Barros et al. 1983; Piazza et al. 2016) y diferente desarrollo de los suelos (del Valle 1998; Cremona et al. 2011). El sitio más húmedo y cercano a la cordillera se localizó en la Península de Quetrihué (48 km del volcán; PMA: 1900 mm; TMA: 8.7 ${ }^{\circ} \mathrm{C}$ ), donde cenizas de variada granulometría cubrieron totalmente el suelo y gran parte de los estratos de vegetación del sotobosque (7-20 cm sobre el suelo; noviembre 2011). El sitio más seco y alejado al volcán se localizó en la zona del Lago Traful (53 km del volcán; PMA: 1600 mm; TMA: $9.5^{\circ} \mathrm{C}$ ), el cual recibió solo cenizas de calibre fino que generaron una pequeña capa sobre el suelo $(1-4 \mathrm{~cm}$, noviembre 2011) y cubrió parcialmente la vegetación del sotobosque. Los dos sitios presentan distintos niveles de productividad primaria, pero no se diferenciaron en la cobertura aérea del dosel (Traful: $76 \pm 2 \%$; Quetrihué: $86 \pm 2 \%$; Piazza et al. 2016), ni en la calidad química de hojarasca de coihue (relación C:N promedio: Traful: $72.7 \pm 1.8 \%$; Quetrihué: 72.9土2.4\%; Piazza 2016).

En cada sitio, se eligieron dos parcelas que se diferenciaron por la presencia/ausencia histórica de ganado bovino extensivo, al menos, por los últimos 50 años. No se cuenta con estimaciones certeras de cargas animales, en parte por la escasa restricción espacial del ganado. En Quetrihué se seleccionó una parcela poco transitable para el ganado y cuyo acceso también fue restringido por Parques

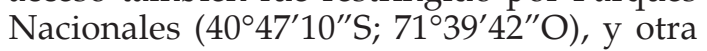
parcela dentro del establecimiento ganadero presente en la península antes de la creación del Parque (4050'16"S; $\left.71^{\circ} 36^{\prime} 43^{\prime \prime O}\right)$. En Traful, se seleccionó una parcela en la isla más grande, donde no había indicios ni registros de ganado $\left(40^{\circ} 35^{\prime} 36^{\prime \prime} \mathrm{S}\right.$; $\left.71^{\circ} 29^{\prime} 56^{\prime \prime} \mathrm{O}\right)$, y otra parcela se ubicó en la península frente a la isla, que es frecuentemente ocupada por ganado asilvestrado $\left(40^{\circ} 35^{\prime} 43^{\prime \prime} S\right.$; $\left.71^{\circ} 29^{\prime} 53^{\prime \prime} \mathrm{O}\right)$. La presencia del ganado en los bosques de coihue altera la estructura de la vegetación, el microambiente y las propiedades del suelo (Piazza et al. 2016; Piazza 2016). En los sitios de estudio se observó que, bajo niveles equivalentes de cobertura del dosel, el ganado redujo un $70 \%$ la cobertura del sotobosque, duplicó la radiación solar incidente e incrementó un 37\% la densidad aparente del suelo (Piazza 2016).

\section{Experimentos de descomposición}

Se realizaron experimentos de descomposición de hojarasca de coihue en los cuatro ambientes definidos por la combinación entre sitio (Quetrihué y Traful) y presencia o ausencia histórica de ganado. La hojarasca fresca se recolectó de las cuatro parcelas, antes de la erupción y libre de cenizas (marzo 2010). En cada sitio se mezclaron las hojas recolectadas en partes iguales de las parcelas con y sin pastoreo, asumiendo un efecto nulo del ganado sobre su calidad foliar (Piazza 2016). Se colocaron $3 \mathrm{~g}$ de hojarasca secada al aire en bolsas de descomposición confeccionadas con mallas de fibra de vidrio (poro $2 \mathrm{~mm}, 15 \mathrm{~cm}$ x $20 \mathrm{~cm}$ ) (Robertson et al. 1999).

La bolsas de hojarasca se incubaron en sus correspondientes sitios $(n=2)$, en 8 bloques distribuidos al azar en un área extensa ( 1 ha) dentro de cada parcela, con o sin pastoreo. Cada bloque contuvo tres bolsas en tres posiciones contiguas: 1) "Bajo ceniza"; las bolsas se colocaron sobre el suelo orgánico y debajo de la capa de cenizas depositada durante 2011. Este tratamiento simuló la posición donde quedó enterrada la hojarasca que cayó antes de la erupción; 2) "Sobre suelo"; diez meses después de la erupción se removió la capa de cenizas para exponer el suelo orgánico a las condiciones micro-climáticas del sitio ( 0.16 $\left.\mathrm{m}^{2}\right)$. Esta manipulación intentó reducir el impacto directo de las cenizas sobre el suelo y evaluar parcialmente el efecto del ambiente de sotobosque sobre la descomposición, y 3) "Sobre ceniza"; las bolsas se colocaron sobre la capa de cenizas sin disturbar y bajo la influencia del ambiente del sotobosque. Este tratamiento representó la condición natural para la hojarasca que se produjo luego de la erupción y que contribuiría a la formación del nuevo horizonte "O" del bosque. La diferenciación visual de la capa de cenizas y la capa de suelo pre-existente fue evidente hasta finalizar el experimento.

Los bloques se protegieron con mallas de alambre para evitar daño por animales. Los experimentos se instalaron en abril 2012 y se cosecharon en marzo 2013. No se realizaron determinaciones intermedias entre dichas fechas. El material remanente se limpió con pincel y se determinó el peso seco libre de cenizas (Robertson et al. 1999); se utilizaron 5 muestras de hojarasca fresca de cada sitio para determinar el contenido de humedad y 
cenizas iniciales. En marzo 2013 se determinó la temperatura (termómetro digital, $2 \mathrm{~cm}$ profundidad), la humedad y el contenido de materia orgánica de la capa de suelo o de ceniza bajo cada bolsa de descomposición para describir la variación espacial entre microambientes, en cada posición y sitio. La humedad gravimétrica $\left(100^{\circ} \mathrm{C}\right.$; 48 horas) y el contenido de materia orgánica total $\left(500^{\circ} \mathrm{C}\right.$; 4 horas) (Robertson et al. 1999) se estimaron a en muestras de la capa de cenizas y de suelo (sin cenizas), de $5 \mathrm{~cm}$ de profundidad y $4 \mathrm{~cm}$ de diámetro. Para diferenciar los sustratos, la profundidad de muestreo se restringió a la profundidad de la capa de cenizas en los experimentos de Traful.

\section{Análisis de los datos}

Para estimar la descomposición, se calculó la pérdida de masa de la hojarasca libre de agua y cenizas al final del experimento respecto a la inicial, luego de un año de incubación a campo. Los resultados se analizaron con modelos lineales mixtos jerárquicos. Dado que el diseño estuvo limitado para evaluar en un único modelo todas las interacciones entre los factores posición, sitio y pastoreo, se analizaron los resultados mediante tres enfoques. Modelo 1: efectos fijos del sitio (2 niveles) y la posición de incubación (3 niveles), con ordenada al origen aleatoria de los efectos anidados de los bloques en parcelas con y sin pastoreo $\left[\mathrm{y}_{\mathrm{i}} \sim \mathrm{N}\left(\mu_{\mathrm{i}}, \sigma_{\varepsilon}^{2}\right) ; \mu_{\mathrm{i}}=\beta_{0 ;[\mathrm{i}]} ; \beta_{0 \mathrm{j}[\mathrm{i}]} \mathrm{N}\left(\mu_{\mathrm{i}}, \sigma_{\mathrm{j}}^{2}\right)\right.$; $\mu_{i}=\alpha_{0}+\alpha 1$.Sitio $+\alpha 2$.Posición $+\alpha 2$.SitioxPosición; $\mathrm{i}=1-32$ bloques, $\mathrm{j}=1-4$ parcelas] (Pinheiro and Bates 2000). Modelo 2: para cada parcela por separado, efecto de la posición anidado por bloque. Modelo 3: para cada sitio (Quetrihué o Traful), se evaluó el efecto de la posición, condición de pastoreo y la interacción, con efecto anidado para 8 bloques en parcelas con y sin pastoreo. Este último enfoque de pseudorepeticiones restringió la interpretación del efecto causal del pastoreo.

Los efectos fijos se evaluaron mediante una prueba F con grados de libertad considerando el anidamiento (Pinheiro and Bates 2000). Se corroboraron los supuestos de normalidad, independencia y homogeneidad de los residuales. Cuando fue necesario se utilizó una estructura de varianza constante para cada bosque o posición [varIdent; $\varepsilon_{\mathrm{jp}} \sim \mathrm{N}(0$, $\left.\sigma_{p}^{2}\right)$ ] (Pinheiro and Bates 2000). Los análisis se realizaron con el paquete nlme, software R (v.2.13.0, Pinheiro et al. 2016; R Core Team 2016). La prueba de Tukey se realizó con el paquete lsmeans (Lenth 2016). Para evaluar la asociación de la descomposición en la hojarasca con las diferencias espaciales de materia orgánica, humedad y temperatura de sustratos donde se incubaron las muestras (suelo o capa de cenizas), se construyó un modelo para cada variable ambiental (efecto fijo) con la estructura anidada aleatoria (sitio/parcela/bloque). La bondad de ajuste se evaluó con los valores de pseudo- $\mathrm{r}^{2}$ marginal, de los efectos fijos, y los valores de pseudo- ${ }^{2}$ condicional, del conjunto de efectos fijos y aleatorios (Nakagawa and Schielzeth 2013; paquete piecewiseSEM, v.1.0.0; Lefcheck 2015). Este análisis no incluyó la heterogeneidad temporal de las variables.

\section{Resultados}

\section{Condiciones microambientales}

Luego de casi dos años de la erupción, la capa de cenizas sobre el suelo presentó valores muy bajos de materia orgánica (promedio 4\%, en las cuatro parcelas), en contraste con la capa superior de suelo del bosque, que en promedio fue del 20\% (Tabla 1). La diferencia entre la capa de cenizas y la capa de suelo orgánico (cubierto y descubierto) fue consistente en los sitios de Quetrihué y Traful (Tabla 2). No se observó una interacción del contenido de materia orgánica entre posiciones con la condición de pastoreo (Tabla 3). El contenido de materia orgánica fue menor en los suelos de Quetrihué (sitio más húmedo) con ganado, respecto de los suelos sin ganado, y se observó el patrón inverso en los bosques de Traful (sitio más seco) (Tabla 1, Tabla 3). A nivel de sitio, la humedad de la capa de cenizas fue menor que en la capa superficial de suelo cubierto o descubierto (Tabla 2, efecto Posición), pero a nivel parcela el efecto solo resultó evidente en Quetrihué sin ganado (Tabla 1, Tabla 3). La temperatura tuvo un patrón variable entre parcelas (Tablas 1, 2, 3). En la parcela de Quetrihué sin ganado, la capa de cenizas presentó $1^{\circ} \mathrm{C}$ menos que la capa de suelo orgánico, pero en la parcela con ganado se observó el patrón inverso. En Traful no se observaron diferencias de temperatura entre capas del suelo (Tabla 1).

\section{Descomposición de hojarasca}

La descomposición dependió de la parcela de bosque y de la posición vertical en el suelo donde se incubaron las muestras (Figura 1, Tabla 2). La hojarasca en contacto con el suelo orgánico, tanto cubierta o no por la capa de cenizas, presentó mayor descomposición 
Tabla 1. Caracterización de los sustratos $(0-5 \mathrm{~cm}$ profundidad, media \pm E.E., $\mathrm{n}=8)$ sobre los que se incubó la hojarasca de $N$. dombeyi en los experimentos de descomposición en bosques situados cerca (1) y lejos (2) del Complejo Volcán Puyehue-Cordón Caulle, y en parcelas sin (a) y con (b) presencia histórica de ganado vacuno.

Table 1. Soil substrate characteristics (0-5 cm depth, mean \pm S.E., $\mathrm{n}=8)$ underneath $N$. dombeyi litterbags used for decomposition experiments in forests located near (1) and far (2) from the Puyehue-Cordón Caulle volcanic system, and plots without (a) and with (b) historical livestock presence.

\begin{tabular}{lllll}
\hline & $\begin{array}{l}\text { 1a. Quetrihué } \\
\text { Sin ganado }\end{array}$ & $\begin{array}{l}\text { 1b. Quetrihué } \\
\text { Con ganado }\end{array}$ & $\begin{array}{l}\text { 2a. Traful } \\
\text { Sin ganado }\end{array}$ & $\begin{array}{l}\text { 2b. Traful } \\
\text { Con ganado }\end{array}$ \\
\hline $\begin{array}{llll}\text { Materia orgánica (\%) } \\
\text { Bajo ceniza }\end{array}$ & $26.8 \pm 9.4 \mathrm{~b}$ & $13.6 \pm 2.0 \mathrm{~b}$ & $13.8 \pm 1.4 \mathrm{~b}$ & $24.8 \pm 6.8 \mathrm{ab}$ \\
$\begin{array}{l}\text { Sobre suelo } \\
\text { Sobre ceniza }\end{array}$ & $31.3 \pm 7.3 \mathrm{~b}$ & $13.0 \pm 2.9 \mathrm{~b}$ & $14.8 \pm 1.3 \mathrm{~b}$ & $22.8 \pm 3.7 \mathrm{~b}$ \\
ANOVA Posición & $\mathrm{F}_{2,14}=7.00 \pm 0.00 \mathrm{a}$ & $0.9 \pm 0.1 \mathrm{a}$ & $5.4 \pm 1.2 \mathrm{a}$ & $10.8 \pm 1.6 \mathrm{a}$ \\
Humedad (\%) & & $\mathrm{F}_{2,14}=14.03, P=0.0005$ & $\mathrm{~F}_{2,14}=19.59, P=0.0001$ & $\mathrm{~F}_{2,14}=7.67, P=0.006$ \\
Bajo ceniza & $34.3 \pm 3.6 \mathrm{ab}$ & $29.3 \pm 06.8 \mathrm{a}$ & $26.0 \pm 2.1 \mathrm{a}$ & $21.6 \pm 3.8 \mathrm{~b}$ \\
$\begin{array}{l}\text { Sobre suelo } \\
\text { Sobre ceniza }\end{array}$ & $44.0 \pm 5.0 \mathrm{~b}$ & $23.6 \pm 01.7 \mathrm{a}$ & $26.3 \pm 1.6 \mathrm{a}$ & $21.6 \pm 1.4 \mathrm{~b}$ \\
ANOVA Posición & $\mathrm{F}_{2,14}=5.04, P=0.02$ & $\mathrm{~F}_{2,14}=0.40, P=0.68$ & $\mathrm{~F}_{2,14}=0.18, P=0.84$ & $17.8 \pm 1.6 \mathrm{a}$ \\
Temperatura ( $\left.{ }^{\circ} \mathrm{C}\right)$ & & & & $\mathrm{F}_{2,14}=3.61, P=0.05$ \\
Bajo ceniza & $10.7 \pm 0.14 \mathrm{~b}$ & $9.8 \pm 0.08 \mathrm{a}$ & $10.5 \pm 0.13 \mathrm{a}$ & $11.3 \pm 0.27 \mathrm{a}$ \\
Sobre suelo & $10.8 \pm 0.15 \mathrm{~b}$ & $9.8 \pm 0.11 \mathrm{a}$ & $10.6 \pm 0.14 \mathrm{a}$ & $10.9 \pm 0.25 \mathrm{a}$ \\
Sobre ceniza & $9.6 \pm 0.22 \mathrm{a}$ & $10.6 \pm 0.36 \mathrm{~b}$ & $10.4 \pm 0.20 \mathrm{a}$ & $10.9 \pm 0.27 \mathrm{a}$ \\
ANOVA Posición & $\mathrm{F}_{2,14}=13.30, P=0.0006$ & $\mathrm{~F}_{2,14}=3.34, P=0.07$ & $\mathrm{~F}_{2,14}=2.40, P=0.13$ & $\mathrm{~F}_{2,14}=3.36, P=0.06$ \\
\hline
\end{tabular}

Nota: Sustrato del tratamiento “Sobre ceniza": cenizas depositadas en 2011. Sustratos de tratamientos "Sobre suelo" y “Bajo ceniza": suelo orgánico del bosque enterrado bajo las cenizas. Resultados del Modelo estadístico 2 (metodología); letras distintas indican diferencias significativas entre posiciones de cada parcela (Tukey, $P=0.05$ ).

Tabla 2. Resultados del análisis de varianza (ANOVA) para los efectos del sitio (Quetrihué vs. Traful) y la posición vertical de hojarasca de $N$. dombeyi (Modelo 1, metodología).

Table 2. Analysis of variance results for the effect of sites (Quetrihué vs. Traful) and N. dombeyi litter position (Model 1 , methods).

\begin{tabular}{llll}
\hline & Sitio & Posición & Sitio x Posición \\
\hline Materia orgánica sustrato $(\%)$ & $\mathrm{F}_{1,2}=0.1, P=0.82$ & $\mathrm{~F}_{2,60}=37.3, P<0.0001$ & $\mathrm{~F}_{2,60}=1.5, P=0.24$ \\
Humedad sustrato $(\%)$ & $\mathrm{F}_{1,2}=1.5, P=0.34$ & $\mathrm{~F}_{2,60}=4.95, P=0.01$ & $\mathrm{~F}_{2,60}=0.85, P=0.43$ \\
Temperatura sustrato $\left({ }^{\circ} \mathrm{C}\right)$ & $\mathrm{F}_{1,2}=1.11, P=0.40$ & $\mathrm{~F}_{2,60}=1.1, P=0.35$ & $\mathrm{~F}_{2,60}=1.15, P=0.32$ \\
Descomposición $(\%)$ & $\mathrm{F}_{1,2}=15.3, P=0.06$ & $\mathrm{~F}_{2,60}=24.4, P<0.0001$ & $\mathrm{~F}_{2,60}=9.5, P=0.0003$ \\
\hline
\end{tabular}

Tabla 3. Resultados del análisis de varianza (ANOVA) para los efectos de la condición de pastoreo (con vs. sin ganado) y la posición de hojarasca de N. dombeyi en los dos sitios de bosque: Quetrihué y Traful (Modelo 3, metodología).

Table 3. Analysis of variance results for the effects of grazing condition (with vs. without livestock), and N. dombeyi litter position in two forest sites: Quetrihué and Traful (Model 3, methodology).

\begin{tabular}{llll}
\hline & Ganado & Posición & Ganado x Posición \\
\hline 1. Quetrihué & & & \\
Materia orgánica sustrato (\%) & $\mathrm{F}_{1,14}=6.18 ; P=0.026$ & $\mathrm{~F}_{2,28}=18.07 ; P<0.0001$ & $\mathrm{~F}_{2,28}=1.78 ; P=0.19$ \\
Humedad sustrato (\%) & $\mathrm{F}_{1,14}=39.73 ; P<0.0001$ & $\mathrm{~F}_{2,28}=5.04 ; P=0.01$ & $\mathrm{~F}_{2,28}=3.95 ; P=0.031$ \\
Temperatura sustrato $\left({ }^{\circ} \mathrm{C}\right)$ & $\mathrm{F}_{1,14}=32.60 ; P=0.0001$ & $\mathrm{~F}_{2,28}=0.82 ; P=0.45$ & $\mathrm{~F}_{2,28}=12.21 ; P=0.0002$ \\
Descomposición (\%) & $\mathrm{F}_{1,14}=13.21 ; P=0.003$ & $\mathrm{~F}_{2,28}=24.29 ; P<0.0001$ & $\mathrm{~F}_{2,28}=2.44 ; P=0.11$ \\
2. Traful & $\mathrm{F}_{1,14}=8.28 ; P=0.012$ & $\mathrm{~F}_{2,28}=22.56 ; P<0.0001$ & $\mathrm{~F}_{2,28}=0.37 ; P=0.69$ \\
Materia orgánica sustrato (\%) & $\mathrm{F}_{1,14}=4.56 ; P=0.051$ & $\mathrm{~F}_{2,28}=1.25 ; P=0.31$ & $\mathrm{~F}_{2,28}=0.15 ; P=0.86$ \\
Humedad sustrato $(\%)$ & $\mathrm{F}_{1,14}=1.17 ; P=0.30$ & $\mathrm{~F}_{2,28}=2.26 ; P=0.12$ & $\mathrm{~F}_{2,28}=4.11 ; P=0.027$ \\
Temperatura sustrato $\left({ }^{\circ} \mathrm{C}\right)$ & $\mathrm{F}_{1,14}=26.61 ; P=0.0001$ & $\mathrm{~F}_{2,28}=2.42 ; P=0.107$ & $\mathrm{~F}_{2,28}=0.92 ; P=0.41$ \\
Descomposición $(\%)$ & & &
\end{tabular}




\section{Quetrihué}

a. Sin Ganado

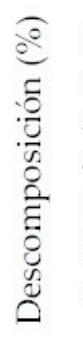

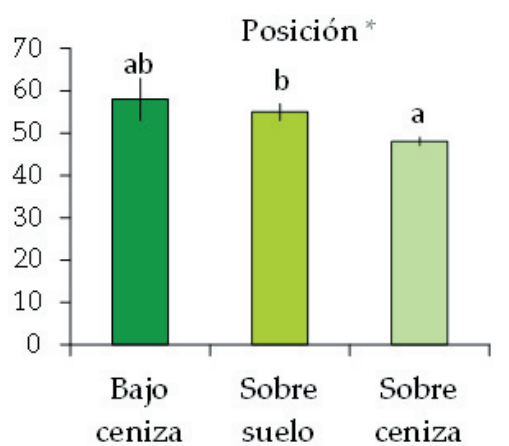

Traful

c. Sin Ganado

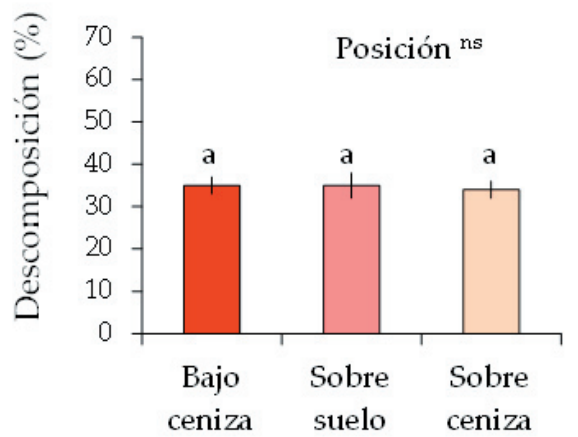

b. Con Ganado

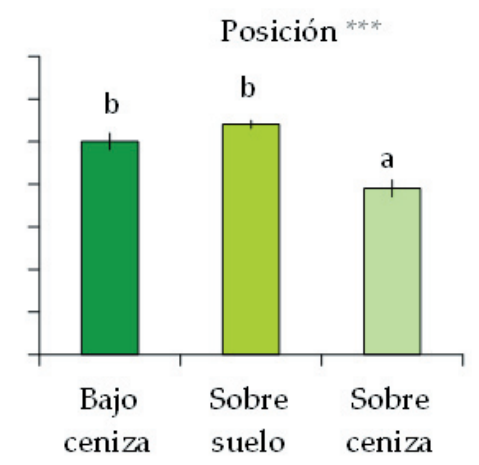

d. Con Ganado

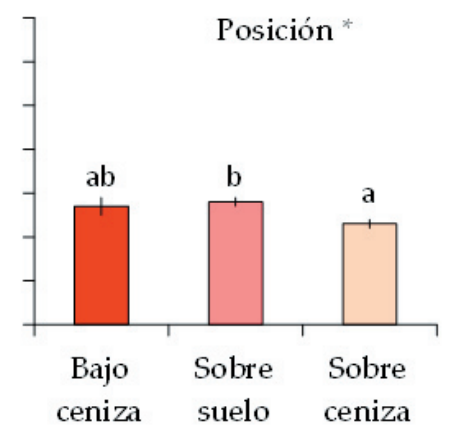

Figura 1. Masa de la hojarasca de N. dombeyi desaparecida (\%) luego de un año de descomposición a campo. Promedio y E.E. ( $\mathrm{n}=8$ ) para cada posición, en los sitios de Quetrihué $(\mathrm{a}-\mathrm{b})$ y Traful $(\mathrm{c}-\mathrm{d})$, y parcelas sin $(\mathrm{a}-\mathrm{c})$ y con $(\mathrm{b}-\mathrm{d})$ influencia histórica de ganado. En cada panel se resume el efecto de la posición de hojarasca en cada parcela (Modelo 2, metodología): ${ }^{* * *} P<0.0001 ;{ }^{*} P<0.05 ;{ }^{\text {ns }} P>0.05$. Letras distintas indican diferencias significativas entre posiciones (Tukey, $\mathrm{p}=0.05$ ).

Figure 1. Litter mass loss (\%) of N. dombeyi after one year of field incubation. Average and S.E. ( $\mathrm{n}=8)$ in Quetrihué (ab) and Traful (c-d) sites, and plots without (a-c) and with livestock historical influence (b-d). Each panel shows effect of litter position in each plot (Model 2, methods): ${ }^{* * *} P<0.0001$; ${ }^{*} P<0.05$; ${ }^{\text {ns }} P>0.05$. Lowercase letters within columns indicate significant differences between positions (Tukey, $\mathrm{p}=0.05$ ).

en las parcelas de Quetrihué que en las de Traful (74\% diferencia). Las diferencias de descomposición entre sitios fueron menores para la hojarasca ubicada sobre la capa de cenizas (52\%; Figura 1). La descomposición luego de un año fue, en promedio para las cuatro parcelas, un $14 \%$ menor para la hojarasca incubada sobre la capa de cenizas que para la hojarasca incubada sobre el suelo orgánico, tanto enterrada por las cenizas o descubierta de cenizas (Figura 1). La diferencia varió en magnitud según el sitio (Tabla 2); el contraste más grande se observó en Quetrihué (12 a $28 \%$; Figura $1 a-b)$, mientras que en Traful las diferencias entre posiciones fueron pequeñas o no significativas según la parcela (Figura 1c-d). En la parcela Quetrihué sin ganado (Figura 1a), la descomposición fue más lenta sobre las cenizas respecto del suelo sin cenizas $\left(\mathrm{F}_{1,7}=8.9, P=0.02\right)$; la posición bajo las cenizas presentó valores de descomposición muy variables $(0.42-0.85)$, por lo que no resultaron diferentes con la posición sobre cenizas $\left(0.47-0.53, \mathrm{~F}_{1,7}=3.5, P=0.10\right)$, ni con la descomposición sobre el suelo sin cenizas $\left(0.50-0.69, \mathrm{~F}_{1,7}=2.5, P=0.12\right)$.

En las parcelas con ganado, la descomposición fue en promedio entre 11 y $25 \%$ menor que en las parcelas sin ganado, para los sitios Quetrihué y Traful, respectivamente (Tabla 3). En general, la influencia histórica de ganado no modificó las diferencias observadas en la descomposición de hojarasca entre posiciones del suelo (Tabla 3, Ganado x Posición: $P>0.05)$. No obstante, al desagregar el análisis en las cuatro parcelas (Figura 1), se observaron diferencias en la magnitud del efecto de la posición vertical de la hojarasca. En la parcela Quetrihué libre de ganado, la 
descomposición sobre las cenizas fue un 13\% menor que para la hojarasca en contacto con el suelo libre de cenizas (Figura 1a), mientras que en Quetrihué con historia de pastoreo, esa diferencia fue mayor (26\%, Figura 1b). En Traful, la descomposición en la parcela con pastoreo fue un $16 \%$ menor sobre las cenizas que sobre el suelo (Figura 1d), mientras que no hubo diferencias significativas entre posiciones del suelo en ausencia de ganado histórico (Figura 1c).

La descomposición de hojarasca (D) se asoció en forma positiva con la materia orgánica del sustrato $(\mathrm{D}=0.37+0.21 \% \mathrm{MO}$; $\mathrm{F}_{1,63}=23.42, \mathrm{p}<0.0001$; pseudo- $\mathrm{r}^{2}$ marginal $/$ condicional $=0.04 / 0.61)$ y con la humedad del sustrato $(\mathrm{D}=0.32+0.29 \%$ Hum; $\mathrm{F}_{1,63}=16.70, P<0.0001$; pseudo- $\mathrm{r}^{2}$ marginal / condicional $=0.04$ / 0.56). Sin embargo, en ambos casos, la proporción de varianza explicada fue pequeña. No se observó asociación entre la descomposición y la temperatura superficial del sustrato $\left(\mathrm{F}_{1,63}=2.02\right.$, $P=0.16)$.

\section{DISCUSIÓN}

En acuerdo con las dos primeras predicciones, en general, la descomposición delahojarasca de $N$. dombeyi fue más lenta cuando fue incubada sobre la capa de cenizas, que bajo las cenizas y en contacto con el suelo orgánico. Ese efecto fue más evidente en los bosques más húmedos y cercanos a la cordillera, que tuvieron mayor impacto por la erupción volcánica. La historia de pastoreo no modificó esas diferencias en descomposición entre posiciones verticales en el suelo, en contradicción con la tercera predicción. Sin embargo, la descomposición de las tres posiciones fue más rápida en las parcelas de $N$. dombeyi sin historia de ganadería que en las parcelas con pastoreo histórico de vacunos. Pese a los efectos de la erupción volcánica en otros procesos, como el crecimiento radial de árboles, la caída de productividad foliar y de la herbivoría del dosel (Chaneton et al. 2014; Magnin et al. 2016), la descomposición de la broza se mantuvo en valores comparables a los registrados en bosques sin influencia de cenizas (Vivanco and Austin 2008; Oleiro 2015). Además, la descomposición en suelos enterrados por cenizas fue equivalente a la descomposición sobre el suelo donde se retiró manualmente la capa de cenizas. Es posible que la restitución del proceso de descomposición de la hojarasca depositada sobre la capa de material volcánico sea más lenta, especialmente en sitios cercanos al volcán, al menos en lo que respecta a la degradación de hojarasca de la especie arbórea dominante.

Los valores de descomposición en nuestros experimentos se situaron dentro del rango registrado en bosques andinos no afectados recientemente por cenizas volcánicas. La descomposición de hojarasca sobre suelos que fueron cubiertos por cenizas fue, en promedio, de $43 \pm 5 \%$ (pérdida de peso). A escala regional, incluyendo bosques de coihue con diferentes ambientes húmedos y secos (1500-2800 mm/año) (Piazza 2016), la descomposición promedio fue de $34 \pm 2 \%$. Previo a la erupción del volcán, en el Parque Nacional Lanín se observó una descomposición anual de coihue alrededor de 33\% (Vivanco and Austin 2008) y $20 \%$ en bosques de la VIII región de Chile (Decker and Boerner 2006); en ambos casos, para bosques situados a mayor elevación y en áreas más secas que nuestros sitios. Además, en un bosque de N. pumilio (Paso Córdoba, $800 \mathrm{~mm} /$ año) la descomposición fue más lenta bajo las cenizas respecto a valores previos a la erupción del CVPCC (Oleiro 2015). No obstante, la escasez de datos para bosques más húmedos y la falta de "testigos reales" por la ausencia de áreas cercanas que no fueron impactadas por el volcán (Chaneton et al. 2014; Magnin et al. 2016) limitan las comparaciones adecuadas y generalizaciones sólidas sobre el efecto de las cenizas en ecosistemas boscosos.

Nuestros resultados mostraron, en general, menor descomposición de la hojarasca de coihue ubicada sobre las cenizas respecto sobre el suelo orgánico (Figura 1). Efectos similares se observaron en bosques cercanos al volcán Santa Elena (Edmonds and Erickson 1994; Erickson and Edmonds 1994) y en un bosque de N. pumilio donde la descomposición fue un $75 \%$ menor sobre la capa de cenizas finas que enterrada bajo la misma (Oleiro 2015). La capa de cenizas con bajos contenidos de materia orgánica podría aislar a la hojarasca depositada con posterioridad a la erupción de los pocos detritívoros y descomponedores activos en el suelo (Elizalde 2014; Oleiro 2015; Berenstecher et al. 2016). La escasez de recursos generada por las cenizas también podría estimular la actividad microbiana presente en el suelo enterrado (Berenstecher et al. 2016). A su vez, la descomposición de la hojarasca enterrada puede ser favorecida por una mayor superficie de contacto con el suelo y/o por mayores niveles humedad y temperatura (Edmonds and Erickson 1994; Austin et al. 
2009). Ello podría explicar la detección de valores similares de descomposición sobre las cenizas y sobre el suelo limpio de cenizas, en contraste con el tratamiento enterrado (Edmonds and Erickson 1994; Erickson and Edmonds 1994; Oleiro 2015). En nuestro estudio, la descomposición fue equivalente en los tratamientos sobre el suelo orgánico, tanto cubierto por cenizas como libre de cenizas, y, en general, ambos difirieron del tratamiento sobre las cenizas (Figura 1). Es posible que los descomponedores del suelo hayan resultado poco sensibles a la deposición de cenizas (cf. Berenstecher et al. 2016), o que nuestros tratamientos de remoción no hayan causado una diferencia ambiental respecto al suelo enterrado, o bien que la presencia o ausencia de la capa superior de cenizas haya inducido una combinación de efectos bióticos / abióticos que haya anulado las diferencias en descomposición.

La descomposición sobre el suelo orgánico reflejó las diferencias ambientales entre los dos sitios de bosque estudiados (Quetrihué vs. Traful), dada su equivalencia en calidad de hojarasca (Piazza 2016). La mayor descomposición sobre el suelo en Quetrihué vs. Traful reflejaría las diferencias de humedad ambiental por su posición geográfica. Por otro lado, la descomposición de hojarasca sobre las cenizas tendió a homogeneizar las diferencias entre bosques (Figura 1). Análogamente, Chaneton et al. (2014) observaron que las cenizas borraron transitoriamente las diferencias funcionales entre bosques húmedos y secos de N. pumilio, al disminuir la productividad foliar en bosques húmedos y reducir la folivoría de insectos en los bosques secos. Si bien la descomposición sobre las cenizas fue relativamente más lenta, los valores de pérdida de masa registrados en esa posición fueron igualmente importantes (30-40\% en un año). Dado el bajo contenido de materia orgánica de la capa de cenizas, la descomposición sobre la misma estaría determinada por factores abióticos (e.g., fotodegradación), y/o por la actividad de organismos endófitos de las hojas o colonizadores ante la limitación de recursos luego de la erupción (Lennon and Jones 2011; Berenstecher et al. 2016).

En bosques maduros de coihue, el pastoreo histórico de ganado modificó el sotobosque al reducir la cobertura vegetal, aumentar la radiación solar incidente y compactar el suelo superficial (Piazza et al. 2016). La tercera predicción postuló que la deposición de cenizas afectaría la descomposición principalmente en bosques cuya estructura no ha sido alterada por la presencia de pastoreo. Bajo dicha lógica, la deposición de cenizas no impactaría la estructura del sotobosque que ya fue alterada por el ganado histórico, limitando la interacción sinérgica entre la cobertura de cenizas y el efecto del pastoreo (del Moral and Grishin 1999). En cambio, los resultados mostraron que la descomposición fue más lenta en bosques con ganado histórico y en todas las posiciones verticales del suelo (Tabla 3). La descomposición sobre las cenizas fue más lenta que sobre el sustrato orgánico, pero esta diferencia fue equivalente en parcelas con y sin pastoreo. Esto sugiere que los mecanismos que desaceleraron la descomposición de broza por las cenizas y por la acción del pastoreo fueron independientes, $\mathrm{o}$ bien que la ausencia de diferencias fue limitada por la falta de repeticiones experimentales. Adicionalmente, los cambios direccionales por la historia de pastoreo sobre la calidad del conjunto de hojarasca (Wardle and Bardgett 2004; Piazza 2016) podría promover efectos sinérgicos de las cenizas con la capa de suelo orgánico enterrado (Dahlgren et al. 2004). Futuros estudios que amplíen el espectro de mecanismos bióticos y abióticos permitirían evaluar con mayor detalle las implicancias de la historia de pastoreo en suelos impactados por eventos volcánicos.

Los resultados de nuestro estudio pueden aportar información preliminar para prever las implicancias de una erupción volcánica en la dinámica de la vegetación. Por un lado, la degradación de la hojarasca se mantuvo activa, lo cual podría asegurar el suministro de nutrientes para las plantas supervivientes al disturbio y/o plantas que logran rebrotar posteriormente (Veblen et al. 2016). Por otro lado, la descomposición más lenta de hojarasca sobre las cenizas sugiere que la formación de nuevo sustrato orgánico y la provisión de nutrientes podrían ser limitantes para el reclutamiento de plántulas. La inmovilización y la estabilización de nutrientes en la matriz física del suelo puede ser mayor en un sustrato inerte e insaturado, como el aportado por las cenizas, respecto al suelo maduro con una comunidad microbiana abundante (Hopkins et al. 2007; Castellano et al. 2015; Cotrufo et al. 2015). Una lenta liberación de nutrientes en el sustrato volcánico podría favorecer a especies de plántulas tolerantes al déficit nutricional, como se ha observado para Nothofagus spp. (Veblen et al. 2016). Siguiendo este razonamiento, en los años posteriores a la 
erupción podrían verse favorecidas especies tolerantes a bajos niveles de nutrientes durante el establecimiento y especies rebrotantes, como arbustos del sotobosque capaces de explorar horizontes bajo las cenizas (Veblen et al. 2016). Este efecto podría ser mayor en sitios con pastoreo, donde la descomposición se vería enlentecida por los cambios en el ambiente y la composición del sotobosque.

En conclusión, este trabajo mostró que la reorganización de la descomposición del bosque luego de una erupción volcánica puede ser diferente en el suelo enterrado, respecto de la nueva capa de sustrato generada por las cenizas. Esas diferencias podrían tener implicancias para la vegetación que recoloniza los horizontes superficiales y que se mantiene activa bajo el impacto de las cenizas (Veblen et al. 2016). No obstante, el proceso de descomposición no se vería interrumpido, reafirmando la existencia de cierta resiliencia en ecosistemas con disturbios relativamente frecuentes durante su historia evolutiva (del Moral and Grishin 1999; Dahlgren et al. 2004). El efecto de enterrado de la hojarasca por las cenizas, sumado a los diferentes contextos ambientales y a cambios inducidos por grandes herbívoros en calidad de la hojarasca que ingresa al suelo, podrían tener efectos complejos para el reciclado de $\mathrm{C}$ y nutrientes en horizontes profundos (Hopkins et al. 2007; Hatton et al. 2014; Cotrufo et al. 2015). Aunque las cenizas y el pastoreo puedan desacelerar la descomposición de materia orgánica, la naturaleza transitoria de ésos efectos sugiere que el funcionamiento del bosque tendría una recuperación relativamente rápida luego de eventos masivos de cenizas volcánicas.

Agradecimientos. Agradecemos al Parque Nacional Nahuel Huapi por la asistencia logística y técnica, especialmente a S. Seijas, L. Pussetto y J. Brunet. A los dos revisores anónimos y a la editora N. Pérez Harguindeguy por sus valiosos comentarios. A M. Oleiro, L. Garibaldi y M. Aguiar por sus contribuciones al proyecto; D. Irsúa, W. de Nicoló, A. Millapán y C. Piazza por su asistencia técnica y P. Pinto por su revisión. Financiación por FONCYT (BID-PICT Redes 2007-0284 y PICT 2011-0386) y becas de ANPCyT y CONICET.

\section{REFERENCIAS}

Antos, J. A., and D. B. Zobel. 2005. Plant responses in forests of the tephra-fall zone. Pages 47-58 in V. H. Dale, F. J. Swanson, and C. M. Crisafulli, editors. Ecological Responses to the 1980 Eruption of Mount St. Helens. Springer, New York. DOI: 10.1007/0-387-28150-9.

Austin, A. T., P. I. Araujo, and P. E. Leva. 2009. Interaction of position, litter type, and water pulses on decomposition of grasses from the semiarid Patagonian steppe. Ecology 90:2642-2647. DOI: 10.1890/08-1804.1

Barros, V. R., V. H. Cordon, C. L. Moyano, R. J. Méndez, J. C. Forqera, and O. Pizzio. 1983. Cartas de precipitación de la zona oeste de las provincias de Río Negro y Neuquén. CONICET, Buenos Aires.

Berenstecher, P., D. Gangi, A. González-Arzac, M. L. Martínez, E. J. Chaves, E. A. Mondino, and A. T. Austin. 2016. Litter microbial and soil faunal communities stimulated in the wake of a volcanic eruption in a semi-arid woodland in Patagonia, Argentina. Functional Ecology 31:245-259. DOI:10.1111/1365-2435.12683

Castellano, M. J., K. E. Mueller, D. C. Olk, J. E. Sawyer, and J. Six. 2015. Integrating plant litter quality, soil organic matter stabilization, and the carbon saturation concept. Global Change Biology 21:3200-3209. DOI: 10.1111/gcb.12982.

Chaneton, E. J., N. Mazía, L. A. Garibaldi, J. Chaij, and T. Kitzberger. 2014. Impact of volcanic ash deposition on foliar productivity and insect herbivory in northern Patagonia deciduous forests. Ecología Austral 24:64-74. DOI: 10.1007/BF00033208.

Cotrufo, M. F., J. L. Soong, A. J. Horton, E. E. Campbell, M. L. Haddix, D. H. Wall, and W. J. Parton. 2015. Formation of soil organic matter via biochemical and physical pathways of litter mass loss. Nature Geoscience 8:776-779. DOI: $10.1038 /$ ngeo2520.

Cremona, V., J. Ferrari, and S. López. 2011. Las cenizas volcánicas y los suelos de la región. Revista Presencia: 8-11.

Dahlgren, R. A., M. Saigusa, and F. C. Ugolini. 2004. The nature, properties and management of volcanic soils. Advances in Agronomy 82:113-182. DOI: 10.1016/S0065-2113(03)82003-5.

Dale, V. H., C. M. Crisafulli, and F. J. Swanson. 2005. 25 years of ecological change at Mount St. Helens. Science 308: 961-2. DOI: 10.1126/science.1109684.

Decker, K. L. M., and R. E. J. Boerner. 2006. Mass loss and nutrient release from decomposing evergreen and deciduous Nothofagus litters from the Chilean Andes. Austral Ecology 31:1005-1015. DOI: 10.1111/j.1442-9993.2006.01670.x.

Edmonds, R. L., and H. E. Erickson. 1994. Influence of Mount St. Helens ash on litter decomposition. I. Pacific silver fir needle decomposition in the ash-fall zone. Canadian Journal of Forest Research 24:826-831. DOI: 10.1139/x94-108.

Elizalde, L. 2014. Volcanism and arthropods: a review. Ecología Austral 24:3-16.

Erickson, H. E., and R. L. Edmonds. 1994. Influence of Mount St. Helens ash on litter decomposition. II. Experimental studies with Douglas-fir needles. Canadian Journal of Forest Research 24:832-838. DOI: 10.1139/x94-109.

Gaitán, J., J. Ayesa, F. Umaña, F. Raffo, and D. Bran. 2011. Cartografía del área afectada por cenizas volcánicas en las provincias de Río Negro y Neuquén. S. C. de Bariloche, Argentina. 
González, R., D. Dec, S. Valle, F. Zúñiga, and J. Dörner. 2015. Efecto de cenizas volcánicas del Cordón Caulle sobre parámetros de calidad física en suelos agrícolas del sur de Chile. Agro Sur 43:53-63. DOI: 10.4206/agrosur.2015.v43n207.

Gutschick, V. P., and H. BassiriRad. 2003. Extreme events as shaping physiology, ecology, and evolution of plants: Toward a unified definition and evaluation of their consequences. New Phytologist 160:21-42. DOI: 10.1046/j.14698137.2003.00866.x

Hatton, P. J., C. Castanha, M. S. Torn, and J. A. Bird. 2014. Litter type control on soil C and N stabilization dynamics in a temperate forest. Global Change Biology 21:1358-1367. DOI: 10.1111/gcb.12786.

Hopkins, D. W., L. Badalucco, L. C. English, S. M. Meli, J. A. Chudek, and A. Ioppolo. 2007. Plant litter decomposition and microbial characteristics in volcanic soils (Mt Etna, Sicily) at different stages of development. Biology and Fertility of Soils 43:461-469. DOI: 10.1007/s00374-006-0124-3.

Iglesias, A., A. E. Artabe, and E. M. Morel. 2011. The evolution of Patagonian climate and vegetation from the Mesozoic to the present. Biological Journal of the Linnean Society 103:409-422. DOI: 10.1111/j.1095-8312.2011.01657.x.

Kardol, P., and D. A. Wardle. 2010. How understanding aboveground-belowground linkages can assist restoration ecology. Trends in Ecology and Evolution 25:670-679. DOI: 10.1016/j.tree.2010.09.001

Kasahara, M., S. Fujii, T. Tanikawa, and A. S. Mori. 2016. Ungulates decelerate litter decomposition by altering litter quality above and below ground. European Journal of Forest Research 135:849-856. DOI: 10.1007/s10342-016-09783.

Lefcheck, J. S. 2015. piecewiseSEM: Piecewise structural equation modeling in R for ecology, evolution, and systematics. Methods in Ecology and Evolution 7:573-579. DOI: 10.1111/2041-210X.12512.

Lennon, J. T., and S. E. Jones. 2011. Microbial seed banks: the ecological and evolutionary implications of dormancy. Nature reviews. Microbiology 9:119-130. DOI: 10.1038/nrmicro2504.

Lenth, R. V. 2016. Least-Squares Means: The R Package lsmeans. Journal of Statistical Software 69:1-33. DOI: 10.18637/ jss.v069.i01.

Magnin, A., R. Villalba, C. D. Torres, M. Stecconi, A. Passo, C. M. Sosa, and J. G. Puntieri. 2016. Effect of volcanic ash deposition on length and radial growths of a deciduous montane tree (Nothofagus pumilio ). Austral Ecology 42: 103-112. DOI: 10.1111/aec.12405.

del Moral, R., and S. Y. Grishin. 1999. Volcanic disturbances and ecosystem recovery. Pages 137-155 in L. R. Walker, editor. Ecosystems of disturbed ground. First edit. Elsevier, Amsterdam.

Nakagawa, S., and H. Schielzeth. 2013. A general and simple method for obtaining R2 from generalized linear mixedeffects models. Methods in Ecology and Evolution 4:133-142. DOI: 10.1111/j.2041-210x.2012.00261.x.

Oleiro, M. I. 2015. Efecto de la diversidad funcional de insectos herbívoros en el ciclado de carbono y nitrógeno en bosques andino-patagónicos. Doctor de la Universidad de Buenos Aires en el área Ciencias Biológicas. Universidad de Buenos Aires, Argentina. Pp.105.

Piazza, M. V., L. A. Garibaldi, T. Kitzberger, and E. J. Chaneton. 2016. Impact of introduced herbivores on understory vegetation along a regional moisture gradient in Patagonian beech forests. Forest Ecology and Management 366: 11-22. DOI: 10.1016/j.foreco.2016.01.035

Piazza, M. V. 2016. Impacto de los herbívoros domésticos sobre el reciclado de C y N: cambios mediados por la vegetación y el ambiente. Doctor en Ciencias Agropecuarias. Universidad de Buenos Aires, Argentina. Pp.169.

Pickett, S. T. A., J. Kolasa, J. J. Armesto, and S. L. Collins. 1989. The ecological concept of disturbance and its expression at various hierarchical levels. Oikos 54:129-136. DOI: 10.2307/3565258.

Pinheiro, J. C., D. M. Bates, S. DebRoy, D. Sarkar, and R. C. Team. 2016. nlme: Linear and Nonlinear Mixed Effects Models.

Pinheiro, J. C., and Bates. 2000. Mixed-effects models in S and S-PLUS. Page Springer. Springer, New York. DOI: 10.1016/j.agsy.2005.11.004.

R Core Team. 2016. R: A language and environment for statistical computing. R Foundation for Statistical Computing, Vienna, Austria.

Robertson, G. P., C. D.C., C. S. Bledsoe, and P. Sollins. 1999. Standard soil methods for long-term ecological research. Oxford University Press, New York.

Semmartin, M., M. R. Aguiar, R. A. Distel, A. S. Moretto, and C. M. Ghersa. 2004. Litter quality and nutrient cycling affected by grazing-induced species replacements along a precipitation gradient. Oikos 107:148-160. DOI: 10.1111/ j.0030-1299.2004.13153.x.

Singer, B. S., B. R. Jicha, M. A. Harper, J. A. Naranjo, L. E. Lara, and H. Moreno-Roa. 2008. Eruptive history, geochronology, and magmatic evolution of the Puyehue-Cordón Caulle volcanic complex, Chile. Bulletin of the Geological Society of America 120:599-618. DOI: 10.1130/B26276.1.

Suzuki, M., and E. Ito. 2014. Combined effects of gap creation and deer exclusion on restoration of belowground systems of secondary woodlands: A field experiment in warm-temperate monsoon Asia. Forest Ecology and Management 329:227-236. DOI: 10.1016/J.FORECO.2014.06.028.

Turner, M. G., W. L. Baker, C. J. Peterson, and R. K. Peet. 1998. Factors influencing succession: lessons from large, infrequent natural disturbances. Ecosystems 1:511-523. DOI: 10.1007/s100219900047.

Turner, M. G., and R. H. Gardner. 2015. Landscape ecology in theory and practice: pattern and process. Springer, New York. DOI: 10.1007/978-1-4939-2794-4.

del Valle, H. F. 1998. Patagonian soils: A regional synthesis. Ecologia Austral 8:103-123. 
Veblen, T., M. González, G. Stewart, T. Kitzberger, and J. Brunet. 2016. Tectonic ecology of the temperate forests of South America and New Zealand. New Zealand Journal of Botany 54:223-246. DOI: 10.1080/0028825X.2015.1130726.

Vivanco, L., and A. T. Austin. 2008. Tree species identity alters forest litter decomposition through long-term plant and soil interactions in Patagonia, Argentina. Journal of Ecology 96:727-736.

Wardle, D. A., and R. D. Bardgett. 2004. Human induced changes in large herbivorous mammal density: the consequences for decomposers. Frontiers in Ecology and the Environment 2:145-153. DOI: 10.1890/1540-9295(2004)002[0145: HCILHM]2.0.CO;2.

Zobel, D. B., and J. A. Antos. 1997. A decade of recovery of understory vegetation buried by volcanic tephra from Mount St. Helens. Ecological Monographs 67:317-344. DOI: 10.1890/0012-9615(1997)067[0317:ADOROU]2.0.CO;2. 